\title{
Use of Topical 5-Fluorouracil for Kerato- cystic Odontogenic Tumors of Gorlin-Goltz Syndrome as a New Targeted Therapy: A Case Report and Review of Literature
}

\author{
Hossein Shiran1 ${ }^{1}$ * , SH Shafaei Fard ${ }^{2}$, S Hakimi ${ }^{3}$, A Azari Pour Esfahani ${ }^{1}$ \\ 1- Postgraduate Student, Oral and Maxillofacial Surgery Dept, faculty of Dentistry, Tehran Medical Sciences, Islamic Azad \\ University, Tehran, Iran \\ 2- Associated Professor, Oral and Maxillofacial Surgery Dept , faculty of Dentistry, Tehran Medical Sciences,, Islamic Azad \\ University, Tehran, Iran \\ 3- Associated Professor, Oral and Maxillofacial Surgery Dept, faculty of Dentistry,Medical Sciences University of Zahedan,Iran
}

\begin{tabular}{l}
\hline ARTICLE INFO \\
Article History \\
Received: May 2019 \\
Accepted: Apr 2019 \\
ePublished: Jun 2019 \\
Corresponding author: \\
Hossein Shiran, Post- \\
graduate Student, \\
Department of Oral and \\
Maxillofacial Surgery, \\
Faculty of Dentistry, \\
Islamic Azad University \\
of Medical Sciences, \\
Tehran, Iran
\end{tabular}

\begin{abstract}
Background and Aim: Keratocystic odontogenic tumors (KOT) have a high rate of recurrence, which is higher in patients diagnosed with Gorlin-Goltz syndrome (GGS). Adjunctive therapies, such as fixative chemical solutions, decrease the rate of recurrence after enucleation and peripheral ostectomy but have high morbidity rates. Topical 5-Fluorouracil (5-FU) has been suggested as a new therapy that provides a directed molecular approach to treatment.

Case Presentation: This is a case report of GGS treated using topical 5-FU as an adjunctive material after enucleation and peripheral ostectomy. New bone formation sites were identified in the radiographic follow-up. The patient was followed up for 10 months regularly without any evidence of recurrence.

Conclusion: 5-FIU is an effective and novel targeted treatment for KOTs. Topical application of 5-FU, following enucleation and peripheral ostectomy, effectively treats syndromic KOTs, resulting in normal bony healing with no adverse local or systemic effects.
\end{abstract}

Keywords: Odontogenic Tumors, Basal Cell Nevus Syndrome, Recurrence, 5-fluorouracil, Salicylic acid Drug Combination [Supplementary Concept]

\section{J Res Dent maxillofac Sci 2019;4(4):43-48}

DOI: 10.29252/jrdms.4.4.43

\section{Introduction:}

The Gorlin-Goltz syndrome (GGS) is an autosomal dominant disorder characterized by a predisposition to neoplasms and other developmental abnormalities. Robert James Gorlin and William Goltz described the first case in 1960 with skeletal abnormalities. ${ }^{(1)}$
GGS or nevoid basal cell carcinoma syndrome (NBCCS) is an autosomal dominant condition with high variability in expression. It is a highly penetrant genetic disorder; which is caused by mutations in the patched-1 (PTCH1), which is a tumor suppressor gene located in 9q22, 3-q31. 
GGS is associated with the presence of multiple odontogenic keratocysts (OKC) and basal cell carcinoma (BCC). The OKC, designated by the World Health Organization (WHO) as a keratocystic odontogenic tumor (KOT), is a locally aggressive, cystic jaw lesion with high growth potential and a propensity for recurrence. ${ }^{(1)}$

KOT is a common manifestation of GGS and can be its first sign in young patients. This syndrome has several names such as basal cell nevus syndrome, multiple BCC syndrome, and the fifth phacomatosis. ${ }^{(2)}$

The gene mutation results in loss of control for several genes known to cause many disorders and affects organogenesis, carcinogenesis, and odontogenesis. A specific gene suppressor, called the patched (PTCH) located in the 9q22.3 chromosome, has been identified as a potential cause of GGS. ${ }^{(2,3)}$ Generally, treatment of KOT involves simple enucleation; however, this treatment is not optimal due to high recurrence. Other treatment options include marsupialization, curettage, peripheral ostectomy, use of adjunctive solutions, removal of the overlying mucosa, and resection. ${ }^{(2)}$

\section{Case Presentation:}

A 19-year-old male patient referred to the oral and maxillofacial clinic for treatment of mandibular lesions with the chief complaint of swelling of the lower jaw. The lesion had started as a small swelling that had increased in size over six months. His past medical history was unremarkable, and he was the second child of non-consanguineous parents.

In the extraoral examinations, the swelling was found to be bilateral, firm, and slightly tender (Figure 1).

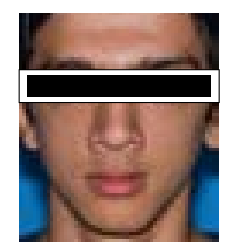

Figure 1. Preoperative photograph of the patient
The panoramic view of the mandible revealed multiple radiolucent lesions on both sides of the mandible and the anterior part of lower jaw. The mandibular lesion on the left side had extended to the ascending ramus of the mandible and was associated with two impacted teeth (\#33 and \#47), which were displaced. Other radiographic examinations were also performed which included chest and skull radiography. Calcification of the falx cerebri and rib abnormality were detected (Figure 2).

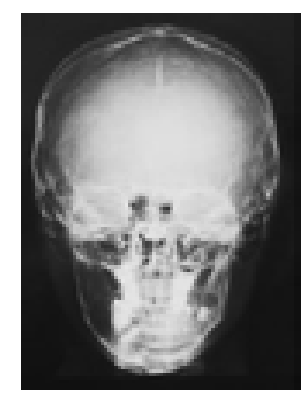

Figure 2. Skull radiograph and calcification of the falx cerebri

Perforation of the buccal plates on the posterior maxilla was detected using cone-beam computed tomography (CBCT; Figure 3).
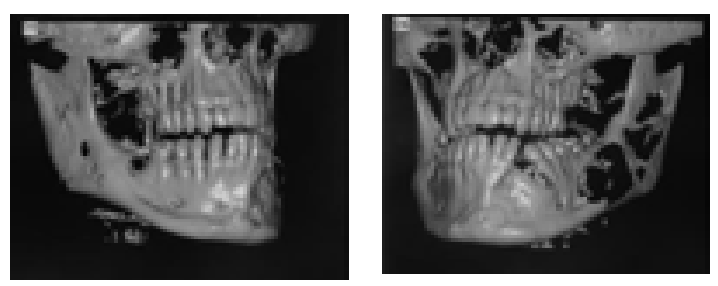

Figure 3. Reconstructed three-dimensional (3D) images from preoperative cone-beam computed tomographic (CBCT) scans

Other clinical examinations revealed dysmorphic facial features including mild macrocephaly, hypertelorism, polydactyly, and palmar and plantar subcutaneous calcifications.

Based on the clinical and radiographic data, the diagnosis of GGS was established.

Decompression of the mandibular lesions was considered as the primary treatment plan. The histopathological evaluation confirmed that the lesions were KOTs. In addition, molecular genetic consultation confirmed PTCH1 germline mutations. 
After 11 months, under general anesthesia, enucleation and surgical removal of the impacted teeth were carried out.

Following peripheral ostectomy of the lesions, a sterile ribbon gauze coated with topical 5-fluorouracil (5-FU, 5\% Efudex Cream, Allergan, Inc., Irvine, CA, USA) was packed into the surgical wounds. ${ }^{(1)}$

The wounds were closed, leaving a small distal end (approximately $1 \mathrm{~cm}$ ) of the gauze exposed to allow gauze removal at 24 hours postoperatively. New bone formation sites were identified in the radiographic follow-up after 3 months due to bone regeneration and remodeling (Figure 4).

The patient was followed up for 18 months regularly without any evidence of recurrence.
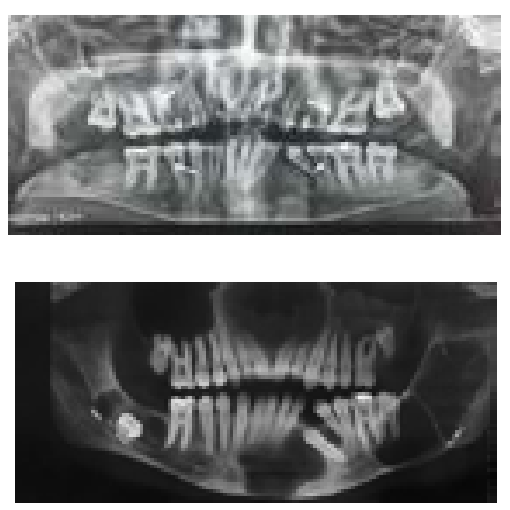

Figure 4. Pre- and postoperative panoramic views
Literature Review:
In the general population, the incidence of GGS is estimated to be 1 in 50,000-150,000. Evans et al reported a minimum prevalence of 1 in 57,000 people. ${ }^{(1)}$ However, a prevalence from $1 / 57,000$ to $1 / 256,000$, with a male to female ratio of $1: 1$ has also been described. Evans et al reported a preva- lence rate of 1/560,000 in the United Kingdom. ${ }^{(1)}$ The syndrome occurs with equal frequency in males and females, but it has mostly been reported in Whites. ${ }^{(2)}$ It has both a sporadic and a familial incidence. Although detected in very young chil- dren, it is commonly expressed between the ages of 17 and 35 years. New mutations are seen in $35 \%$ to $50 \%$ of mutations of PTCH1, which is part of the sonic hedgehog (SHH) signaling pathway and is the molecular basis of the syndrome.

This gene was first isolated in 1996 as the human homolog of the Drosophila segment polarity gene, mapped to the long arm of chromosome 9q22.3-q31 with no apparent heterogeneity in Australia and the United States. This gene plays a role in tumor suppression, embryonic structuring, and cellular cycle. ${ }^{(1)}$ Nine variants of mutations have been reported in patients with GGS. ${ }^{(1)}$

Pruvost-Balland et al carried out a clinical and genetic study on 22 patients with GGS. (4) PTCH 1 mutations were identified in 13 patients, out of which, six were familial cases, three were sporadic, and in four patients, it was not possible to conclude if they were familial. ${ }^{(4)}$

Diagnosis of this syndrome may be difficult because of the variability of expressivity and because of different ages of onset for the different traits of this disorder. ${ }^{(5)}$ Early diagnosis of GGS is crucial for the affected children and their families, considering the risk of developing malignancies such as medulloblastoma and aggressive skin cancers. ${ }^{(5)}$

A negative family history could hamper the early clinical recognition of patients with GGS. However, it may be diagnosed during early childhood if the clinician is well aware of the clinical signs of the disease. The diagnostic criteria for GGS were introduced by Evans et al (1991) and were modified by Kimonis et al (1997). (1,5) According to Kimonis et al, diagnosis can be established onlywhen two major or one major and two minor criteria are present. (5)

The major criteria are as follows: more than two BCCs or one BCC in patients younger than 20 years of age, OKCs of the jaw (proven by histologic analysis), three or more palmar or plantar pits, bilamellar calcification of the falx cerebri, bifid and fused or markedly splayed ribs, and a first-degree relative with NBCC. ${ }^{(5)}$ The minor criteria are as follows: macrocephaly, congenital malformations, e.g., cleft lip or palate, frontal bossing, coarse faces, and moderate or severe hypertelorism, other skeletal abnormalities, e.g., Sprengel deformity, marked pectus deformity, and marked syndactyly of the 
digits, radiological abnormalities, e.g., bridging of the sella turcica, vertebral anomalies, modeling defects of the hands and feet, or flameshaped lucencies of the hands and the feet, and ovarian fibroma or medulloblastoma. ${ }^{(5)}$

OKCs linked with GGS, now termed as KOTs, are a constant feature present in about $75 \%$ of cases with GGS. ${ }^{(6-8)}$ KOTs are often the first sign of GGS in $78 \%$ of cases. They develop during the first decade of life, usually after 7 years, and peak during the second and third decades. Their occurrence is approximately a decade earlier than that of OKCs not associated with the syndrome. The male to female ratio is 1:0.62 for conventional OKCs and 1: 1 for KOT. ${ }^{(6-8)}$

KOTs have a greater predilection for the mandible $(69 \%)$ than the maxilla $(31 \%){ }^{(6-}$ 8) In the mandible, $43 \%$ of KOTs occur in the molar-ramus region, followed by $18 \%$ in the incisor-canine region, and $7 \%$ in the premolar region. In the maxilla, $14 \%$ of KOTs occur in the incisor-canine region, followed by $12 \%$ in the molar-tuberosity region, and 3\% in the premolar region. ${ }^{(6-8)}$

In young patients, the cysts may be associated with unerupted teeth, causing tooth displacement and root resorption. They are asymptomatic unless secondarily infected and rarely cause pathological fractures. ${ }^{(8)}$

Ameloblastoma and squamous cell carcinoma (SCC) can originate from these cysts. On the panoramic view, KOTs may show a unilocular or multilocular shape, and the cystic spaces may have smooth or scalloped borders. Multiple OKCs may be suggestive of the syndrome. (8)

Histologically, in comparison with conventional OKCs, syndromic KOTs show more satellite cysts, solid islands of epithelial proliferation, intramural epithelial remnants, odontogenic rests within the capsule, and increased parakeratinization and mitotic figures in the epithelium. Moreover, syndromic KOTs have shorter epithelial height and smaller nuclei when compared to solitary OKCs. Immunohistochemical (IHC) analysis has shown that cytokeratins CK17 and CK19 are overexpressed in OKCs. ${ }^{(9)}$
There are two methods of treating OKCs: conservative and aggressive. In the conservative method, simple enucleation with or without curettage and marsupialization is suggested. Aggressive methods include peripheral ostectomy, chemical curettage with Carnoy's solution (CS), and resection. Insertion of CS into the cystic cavity three minutes after enucleation results in a lower rate of recurrence $(0-2.5 \%)$ without damaging the inferior alveolar nerve (IAN). ${ }^{(7)}$ Moreover, the use of CS following cyst enucleation and cryosurgery is advised to destroy epithelial remnants and dental lamina within the osseous margin, thus preventing recurrences. ${ }^{(7)}$ Cryosurgery using liquid nitrogen is indicated in large mandibular lesions if there is a risk of damage to vital structures with conventional treatments ${ }^{(9)}$

En bloc resection of OKCs is considered in the following situations: when cysts recur despite previous enucleation with an adjunctive procedure, when cysts recur despite previous marsupialization and enucleation with an adjunctive procedure, in cases of multilocular aggressive cysts, in cases of multiple nonsyndromic and syndromic cysts, and cysts with aggressive clinical behavior that require resection. ${ }^{(10)}$

The recurrence rate of KOT after excision is high, ranging from $12 \%$ to $62.5 \%$ with multiple recurrences. Recurrence rates of $82 \%$ and $61 \%$ have also been reported for KOT and solitary OKCs. ${ }^{(7)}$

An annual panoramic radiograph is usually recommended between the ages of 8 and 40 years for checking the recurrence or development of new KOTs. A recurring cyst can be a new cyst that originates from epithelial residue or a microcyst in the overlying mucosa. It is believed that the aggressive behavior and high rate of recurrence of KOTs are due to the higher rate of epithelial proliferation. ${ }^{(11)}$

Enucleation results in recurrence rates as high as $56 \%$ while resection results in recurrence rates closer to $0 \%$. The use of Carnoy's solution (absolute alcohol, glacial acetic acid, chloroform, and ferric chloride) decreases the rate of recurrence. ${ }^{(11)}$

Since chloroform is carcinogenic, it has been removed from CS, giving rise to modified Carnoy's solution (MC). It has been shown that the use of $\mathrm{MC}$ is associated with significantly higher 
recurrence rates. ${ }^{(11)}$ Rates of peripheral nerve injury after the use of CS are estimated to be $18.2 \%$ after direct use of the solution. ${ }^{(12,13)}$

A directed approach has been suggested for KOT treatment according to the molecular genetics of KOTs. KOTs and BBCs develop via PTCH gene mutations. Mutations in PTCH1 cause the activation of smoothened (SMO) and SHH signaling, resulting in neoplastic growth. ${ }^{(14)}$ Booms and colleagues demonstrated that SMO gene alterations play an important role in KOT development. ${ }^{(15)}$ This finding suggests that SHH signaling pathway antagonism may be an efficient way to target KOTs. ${ }^{(13,14)}$

5-fluorouracil (5-FU), which is an antimetabolite drug, has been shown to induce apoptosis through inhibiting SHH. 5-FU has several applications in treatment of malignant disease including topical application to treat superficial BCCs. ${ }^{(15,16)}$

The purpose of this report was to determine the efficacy of topical 5\% 5-FU for the treatment of syndromic KOTs. We hypothesized that 5-FU is an effective treatment for this type of KOTs. Topical application of 5-FU after enucleation and peripheral ostectomy treats KOTs, resulting in normal healing with no adverse local or systemic effects. ${ }^{(17)}$

Although topical application of 5-FU has no adverse effects, systemic use of 5-FU may result in adverse reactions including mucositis, granulocytopenia, neuropathy, cardiac toxicities, nausea, vomiting, pallor, hypotension, general malaise, and death. ${ }^{(17)}$

Ledderhof et al conducted a cohort study for treatment of patients with non-syndromic KOTs and compared the topical application of 5-FU with MC solution and suggested 5-FU as a new therapy. There were no KOT recurrences with 5-FU whereas there were recurrences with MC. Also, there was a significantly lower incidence of IAN paresthesia with 5-FU. ${ }^{(18)}$ Supplementary molecular characterization and clinical trials are suggested for this treatment of KOTs.

\section{Conclusion:}

5-FU is an effective and novel targeted treatment for KOTs. Topical application of 5-FU after enucleation and peripheral ostectomy effectively treats syndromic KOTs, resulting in normal bony healing with no adverse local or systemic effects. 5-FU may be ideal due to its availability, ease of use, short operating time, and decreased morbidity. It is also an accessible and well-studied drug.

\section{References:}

1. Evans DG, Ladusans EJ, Rimmer S, Burnell LD, Thakker N, Farndon PA. Complications of the naevoid basal cell carcinoma syndrome: results of a population based study. J Med Genet. 1993 Jun;30(6):460-4.

2. Gu XM, Zhao HS, Sun LS, Li TJ. PTCH mutations in sporadic and Gorlin-syndrome-related odontogenic keratocysts. J Dent Res. 2006 Sep;85(9):859-63.

3. Acocella A, Sacco R, Bertolai R, Sacco N. Genetic and clinicopathologic aspects of GorlinGoltz syndrome (NBCCS): presentation of two case reports and literature review. Minerva Stomatol. 2009 Jan-Feb;58(1-2):43-53.

4. Pruvost-Balland C, Gorry P, Boutet N, Magnaldo T, Mamelle G, Margulis A, et al. [Clinical and genetic study in 22 patients with basal cell nevus syndrome]. [Article in French]. Ann Dermatol Venereol. 2006 Feb;133(2):117-23.

5. Kimonis VE, Goldstein AM, Pastakia B, Yang ML, Kase R, DiGiovanna JJ, et al. Clinical manifestations in 105 persons with nevoid basal cell carcinoma syndrome. Am J Med Genet. 1997 Mar 31;69(3):299-308.

6. Myoung H, Hong SP, Hong SD, Lee JI, Lim $\mathrm{CY}$, Choung PH, et al. Odontogenic keratocyst: Review of 256 cases for recurrence and clinicopathologic parameters. Oral Surg Oral Med Oral Pathol Oral Radiol Endod. 2001 Mar;91(3):32833.

7. Dominguez FV, Keszler A. Comparative study of keratocysts, associated and non-associated with nevoid basal cell carcinoma syndrome. J Oral Pathol. 1988 Jan;17(1):39-42.

8. Woolgar JA, Rippin JW, Browne RM. The odontogenic keratocyst and its occurrence in the nevoid basal cell carcinoma syndrome. Oral Surg Oral Med Oral Pathol. 1987 Dec;64(6):727-30.

9. Stoelinga PJ. The treatment of odontogenic keratocysts by excision of the overlying, attached mucosa, enucleation, and treatment of the bony defect with carnoy solution. J Oral Maxillofac Surg. 2005 Nov;63(11):1662-6.

10. Schmidt BL, Pogrel MA. The use of enu- 
cleation and liquid nitrogen cryotherapy in the management of odontogenic keratocysts. J Oral Maxillofac Surg. 2001 Jul;59(7):720-5; discussion 726-7.

11. Tolstunov L, Treasure T. Surgical treatment algorithm for odontogenic keratocyst: combined treatment of odontogenic keratocyst and mandibular defect with marsupialization, enucleation, iliac crest bone graft, and dental implants. J Oral Maxillofac Surg. 2008 May;66(5):1025-36.

12. Stoelinga PJ. Excision of the overlying, attached mucosa, in conjunction with cyst enucleation and treatment of the bony defect with carnoy solution. Oral Maxillofac Surg Clin North Am. 2003 Aug;15(3):407-14.

13. Gosau M, Draenert FG, Müller S, Frerich B, Bürgers R, Reichert TE, et al. Two modifications in the treatment of keratocystic odontogenic tumors (KCOT) and the use of Carnoy's solution (CS)--a retrospective study lasting between 2 and 10 years. Clin Oral Investig. 2010 Feb;14(1):2734.

14. Zhang L, Sun ZJ, Zhao YF, Bian Z, Fan MW, Chen Z. Inhibition of SHH signaling pathway: molecular treatment strategy of odontogenic keratocyst. Med Hypotheses. 2006;67(5):1242-4. 15. Booms P, Harth M, Sader R, Ghanaati S. Vismodegib hedgehog-signaling inhibition and treatment of basal cell carcinomas as well as keratocystic odontogenic tumors in Gorlin syndrome. Ann Maxillofac Surg. 2015 Jan-Jun;5(1):14-9.

16. Gross K, Kircik L, Kricorian G. 5\% 5-Fluorouracil cream for the treatment of small superficial Basal cell carcinoma: efficacy, tolerability, cosmetic outcome, and patient satisfaction. Dermatol Surg. 2007 Apr;33(4):433-9; discussion 440.

17. Papanastasopoulos P, Stebbing J. Molecular basis of 5-fluorouracil-related toxicity: lessons from clinical practice. Anticancer Res. 2014 Apr;34(4):1531-5.

18. Ledderhof NJ, Caminiti MF, Bradley G, Lam DK. Topical 5-Fluorouracil is a Novel Targeted Therapy for the Keratocystic Odontogenic Tumor. J Oral Maxillofac Surg. 2017 Mar;75(3):514-524.
Please cite this paper as:

shiran H, Shafaei Fard S, Hakimi S, Azari Pour

Esfahani A. Use of Topical 5-Fluorouracil for Keratocystic Odontogenic Tumors of GorlinGoltz Syndrome as a New Targeted Therapy: A Case Report and Review of Literature. J Res Dentomaxillofac Sci. 2019; 4 (4) :43-48 